\title{
Multiband Antennas for SDR Applications
}

\author{
E. Surducan, ${ }^{1,2}$ V. Surducan, ${ }^{1,2}$ D. Iancu, ${ }^{1,3}$ and J. Glossner ${ }^{1}$ \\ ${ }^{1}$ Sandbridge Technologies, 120 White Plains Road, 4th floor Tarrytown, NY 10591, USA \\ ${ }^{2}$ Department of Molecular and Biomolecular Physics, National Institute for Research and Development of \\ Isotopic and Molecular Technologies, 65-103 Donath Street, 400293 Cluj Napoca, Romania \\ ${ }^{3}$ Department of Computer Systems, Tampere University of Technology, Korkeakoulunkatu, 3, 33720 Tampere, Finland
}

Correspondence should be addressed to E. Surducan, esurducan@gmail.com

Received 30 September 2008; Accepted 12 February 2009

Recommended by Mihai Sima

We present multiband antennas configurations for SDR applications. Using a composite folded dipole structure as starting point, we derived more complex antenna configurations to support multiple communication protocols for mobile application with linear and circular polarizations. Prototypes as single antenna with circular polarization, tunable single antenna with PIN diode and MIMO systems with three and four antennas, all derivatives of the same basic structure, were produced in an iterative fashion until the desired parameters were achieved. These antennas are suitable for microstrip circuit realizations and can be included in the printed circuit board (PCB) of the device, or used as stand alone. The shapes and measurement results are presented throughout the paper. From the illustrated graphs it can be seen that the stand-alone antennas exhibit positive gain for all the frequency bands of interest while the separation between antennas, for the multiple-input multiple-output (MIMO) case, is better than $15 \mathrm{~dB}$.

Copyright (C 2009 E. Surducan et al. This is an open access article distributed under the Creative Commons Attribution License, which permits unrestricted use, distribution, and reproduction in any medium, provided the original work is properly cited.

\section{Introduction}

To comply with the requirements of portable multiplecommunication protocol enabled hand-held devices, a multiband antenna solution is highly desired. Most of the existing antennas used in current hand-held devices have negative gain of several $\mathrm{dBs}$ while base-band processing struggles to recover the last tenth of $\mathrm{dB}$ gain through more complex algorithms. However, considering antenna performance requirements and the tradeoffs associated with operation over multiple disparate frequency bands, constraints on size and limitations of commercial lowcost materials, typically required in handset designs, this task becomes very challenging and highly difficult. The multiband antennas configurations presented in this paper are part of the SDR platform developed by Sandbridge Technologies [1].

Ultra-wideband antennas and diversity techniques were intensively investigated and presented in the literature with not only the goal of optimizing the gain and bandwidth [2], but also by optimizing the number and position of antennas in stationary as well as in mobile devices. Multiple-input multiple-output (MIMO) antenna systems and diversity techniques are used successfully in today's wireless communications as part of the effort of increasing the overall data throughput and link reliability [3, 4]. However, for space diversity, one requirement is to place the antennas at least one half wavelength apart. This restriction raises significant challenges when two or more antennas are to be placed on today's hand-held devices. Released and future release standards such as high-speed packed access (HSPA), long-term evolution (LTE), and worldwide interoperability microwave access (WiMAX), all specify open and/or close loop MIMO systems.

In our approach, we started the antenna design with a modified printed dipole antenna as miniature dipoles with split arms, targeting wireless local area network (WLAN) at 2.45 and $5 \mathrm{GHz}$ frequencies bands [5-7]. A dual mode microstrip antenna [8] using a switch for selecting one of the two antennas is described in [9]. To avoid the extra components cost and the associated power consumption, in our solution, we choose two dipoles working on different frequencies and fed by microstrip lines at the same location. This configuration looks like miniature microstrip dipoles with split arms. We applied this technique first to the digital video broadcasting terrestrial/hand-held (DVB-T/H) 

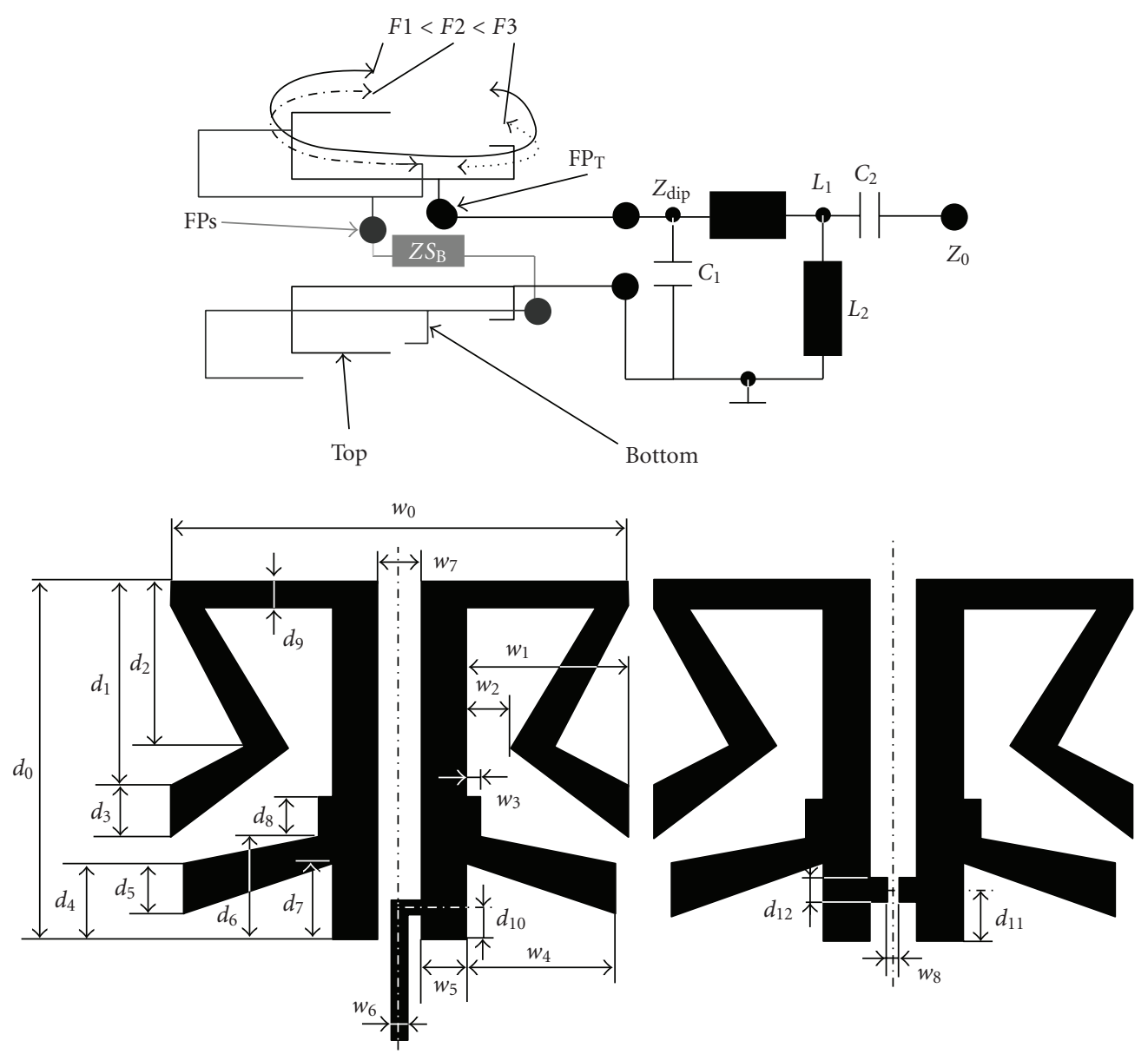

FIgure 1: Schematic of the basic antenna configurations. $L_{1}$ and $C_{1}$ are distributed values due to the PCB and $L_{2}, C_{2}$, and $Z S_{\mathrm{B}}$ are surface mount components; $Z_{0}$ is the line impedance $(50 \mathrm{Ohm})$ and $Z_{\mathrm{dip}} \sim 200 \mathrm{Ohm}$. The $d_{i}$ ) and $w_{i}$ ) dimensions figured on the PCB top and bottom side are specific to the particular antenna design.

frequency bands by using a composite structure, miniature directive antenna with high gain as shown in $[10,11]$, similar to metamaterial dielectric support antennas [12, 13] as a solution to enlarge the frequencies bands. As a second approach, similar to a Yagi antenna applied to microstrip structures [14], we used a selective reflector placed behind the folded dipole on multilayer dielectric substrate in order to enlarge de frequency bands. We have redesigned the composite antenna for multiple communication standard hand-held devices, supporting frequency bands specified by DVB-H, GSM, and WCDMA and we incorporated the antenna to the printed circuit board (PCB) of the device [15]. Early efforts to implement MIMO antenna systems are described in automotive [2], and hand-held devices [10, 11, 16]. Our form factor WLAN and WiMAX antenna design, with two receive and one transmit antennas, is described in [17].

A common solution to tune the multiband antenna is the PIN diode switching technique [18, 19]. Our first effort in adding a PIN diode to our antenna is presented in [20]. The antenna polarization can also be controlled by PIN diodes, as shown later the paper, servicing this way the GPS L2 band.
The paper is structured as follows. Section 2 describes the approach we took for the antennas design, the measurement approach is shown in Section 3 while Section 4 presents practical realization and measurement results for different antennas. Section 5 concludes the paper.

\section{Antenna Design}

The new antenna configurations are based on our previous work on multiband composite antennas supporting DVB and WLAN frequency bands. The basic idea is to start with the composite antenna and add a selective reflector in the near field location of the active antenna (active in the sense of main radiator not in the sense of active devices on the layer). Constructively, it is an antenna with two conductive layers of similar printed patterns, top and bottom, and a composite dielectric substrate of one or more dielectric layers with different permittivity $[10,11]$. On the top side is placed the matching circuit to the microwave line. The equivalent schematic of the matching circuit, and the top and bottom side PCB of the antenna (with related dimensions) are illustrated in Figure 1. $L_{1}$ and $C_{1}$ are distributed values 


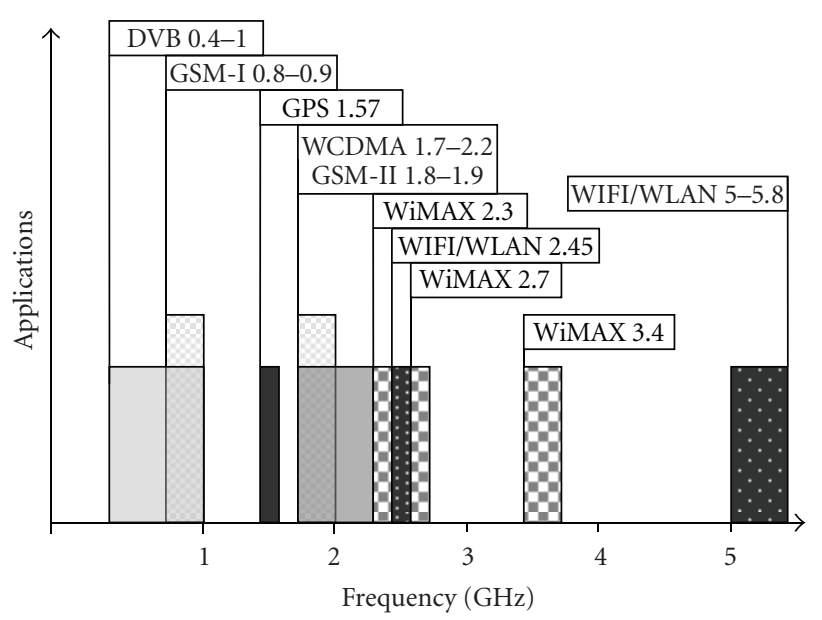

Figure 2: Frequency bands covered with composite antennas.

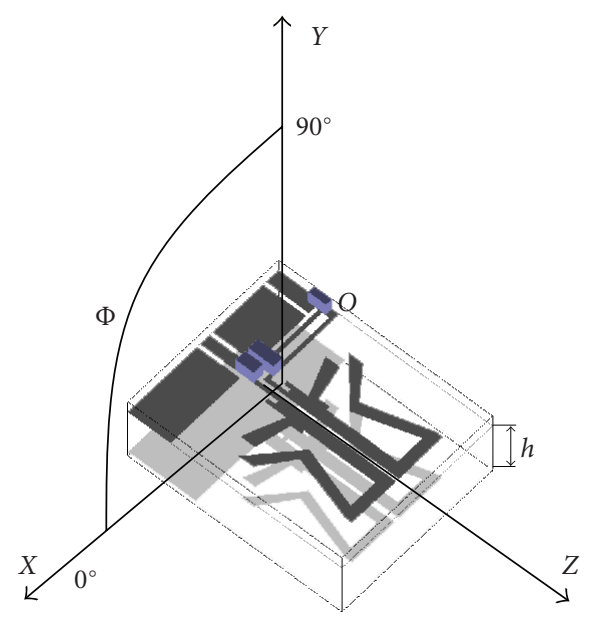

FIgUre 3: TheXOY plan where the directive gain was measured for the experimental antenna.

due to the PCB, $L_{2}, C_{2}$, and $Z S_{B}$ are lumped surface mount components. The line impedance $Z_{0}$ and the dipole impedance $Z_{\text {dip }}$ are 50 and $200 \mathrm{Ohms}$, respectively.

In fact, the antenna is an inductive multifolded dipole structure [5], exhibiting multiple resonant frequencies $F 1<$ $F 2<F 3$, and having a common feed point located at zero voltage and maximum current. In Figure 1, the feed point on the top side is $\mathrm{FP}_{\mathrm{T}}$ and the related feed point on the bottom side is $\mathrm{FP}_{\mathrm{B}}$. The load impedance at the feed point on the reflector side $\mathrm{FP}_{\mathrm{B}}$ may be short or open circuit or, an impedance load, $Z S_{\mathrm{B}}$. The position $\left(d_{10}\right)$ of the feed on the top side $\mathrm{FP}_{\mathrm{T}}$ is crucial for the multiresonance frequencies of the antenna. The electrical properties of the composite dielectric between the two conductive layers are directly related to the gain as well as the width of the frequency bands covered by the antenna. With this antenna configuration it is possible to cover all frequency bands required by a specific application, with little change of the basic pattern. A large variety of antennas have been designed with dimensions ranging from 10 to $25 \mathrm{~mm}$ width, 20 to $50 \mathrm{~mm}$ length,

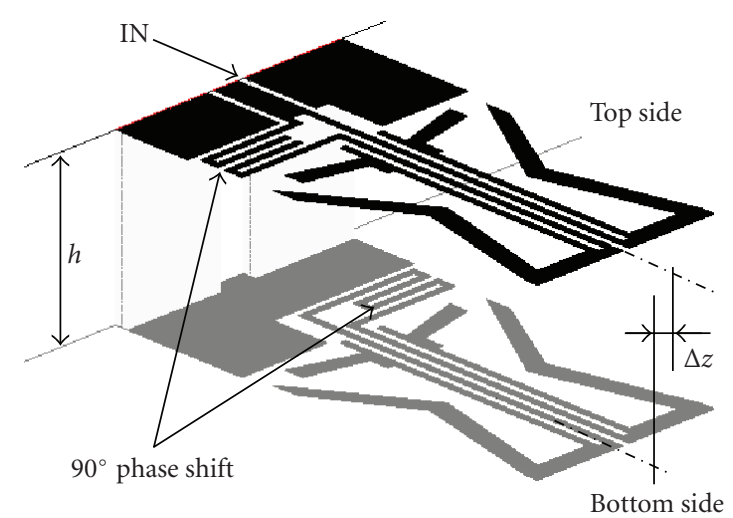

FIGURE 4: GPS omnidirectional antenna.

and PCB thickness from 0.8 to $2.54 \mathrm{~mm}$. The dielectric permittivity $\varepsilon_{r}$ was also varied from 2.8 to 10 . In the following we present only the most representatives of the produced antennas. The range of the frequency bands for different communication standards is illustrated in Figure 2.

\section{Measurements}

The most important parameters characteristic to antennas are the return loss $\left(S_{11}\right)$ or the standing wave ration (SWR), gain and directivity. All measurements were performed using the Agilent N5230A (PNA-L) VectorNetwork Analyzer (VNA). The $S_{11}$ (or SWR) was measured directly with the VNA. Between $S_{11}$ and SWR there is the following well known conversion formula [8]:

$$
\operatorname{SWR}=\frac{1+\left|S_{11}\right|}{1-\left|S_{11}\right|}
$$

For a reasonably good antenna the SWR must be less than 3 .

For the gain calculation, the Friis transmission equation was considered with the far-field condition as described in $[21,22]$ :

$$
P_{R}=\left(\frac{\lambda}{4 \pi R}\right)^{2} G^{2} P_{T}, \quad \text { with } R \geq \frac{2 d^{2}}{\lambda} .
$$

In the above equation, $P_{R}$ is the power measured at the receive antenna output port, $P_{T}$ is the power measured at the transmit antenna input port, $G$ is the gain for both transmit and receive antennas, considered identical, $\lambda$ is the wavelength, $R$ is the separation between antennas, and $d$ is the largest physical dimension of the antenna. From (2), knowing that $S_{12}=S_{21}$, through simple algebraic manipulation, the gain follows:

$$
G=-3.779+10 \log (R F)+0.5\left(S_{21}\right)+2.15,
$$

where $G$ is measured in $\mathrm{dBi}$, the transmission parameter $S_{12}$ in $\mathrm{dB}, R$ in $\mathrm{cm}$, and $F$ in $\mathrm{GHz}$. 


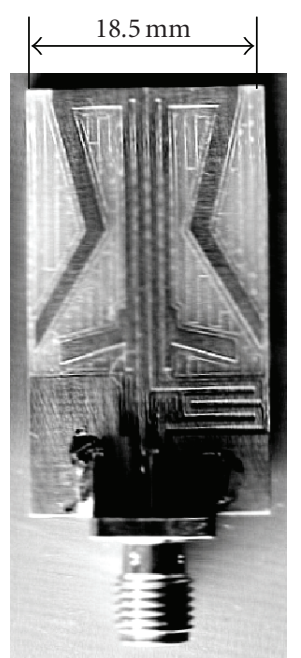

Top

(a)

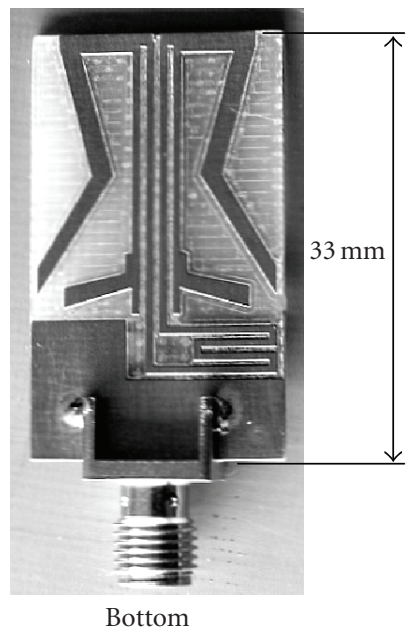

(b)
FIGURE 5: Pictures of GPS antenna, top and bottom sides.

The directivity $D$ is the measure of the directional properties of the antenna related to the isotropic antenna. The directivity gain $G$, related to the isotropic dipole is also defined in [8] as

$$
G=e D, \quad \text { with } 0<e<1,
$$

where $e$ is the radiation efficiency of the antenna.

The directive gain was calculated with relation (3). The $S_{21}$ parameter was measured using two identical antennas or an antennas system. To obtain the directive gain diagram one antenna was rotated around $O Z$ direction as shown in Figure 3. In the case of the antennas system the isolation between antennas was measured directly with the VNA through the transmission parameter the between two antennas considering that $S_{i j}=S_{j i}$.

\section{Antennas}

4.1. GPS Antenna. The GPS satellites transmit right-hand circularly polarized (RHCP) $L$-band signal known as $L 1$ at 1575.42 MHz. The minimum signal power level is $-160 \mathrm{dBw}$ at the Earth's surface. The very low power level of the GPS signal requires passive antennas of a few $\mathrm{dB}$ gains in the immediate proximity of the receiver or, an active antenna of minimum $15 \mathrm{~dB}$ gain if the antenna is placed on the roof of a car, building, or boat. To achieve circular polarization from our antenna configuration, we introduced a $90^{\circ}$ delay line between the two branches of the folded dipole in both sides, top and bottom, as shown in Figure 4.

The top feed is through a microstrip while the bottom feed is inductive. A shift of $\Delta z$ between the top and bottom layer was added, as shown in Figure 4, to compensate for the phase shift between the two sides.

We built two-prototype antennas, first on RT-6010LM substrate: $2.45 \mathrm{~mm}$ thick, $15 \mathrm{~mm}$ wide, $27 \mathrm{~mm}$ high, permittivity $\varepsilon_{r}=10.7$, and dissipation factor $\operatorname{tg} \delta=0.0028$, and the

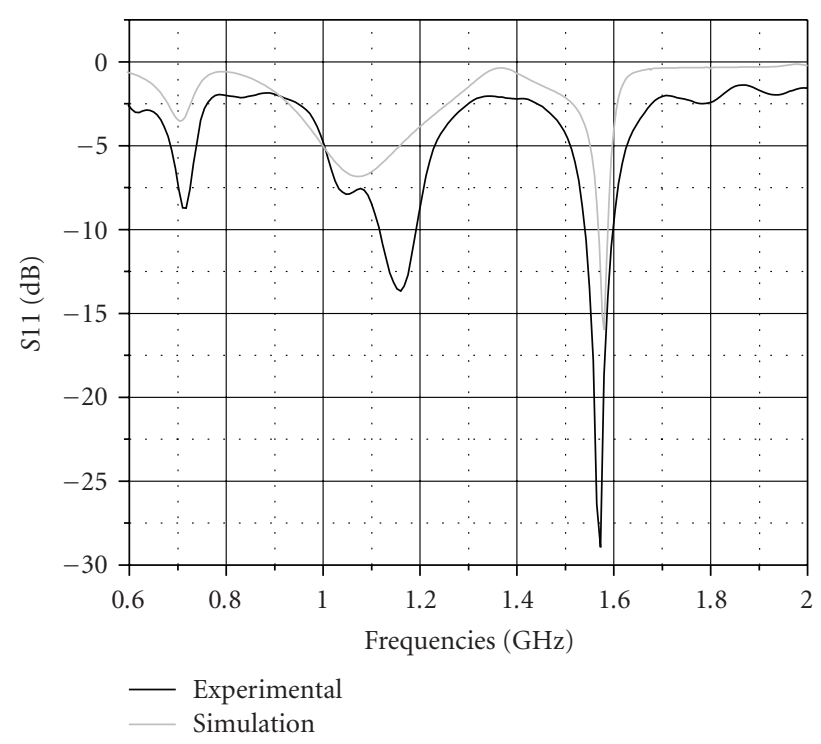

FIGURE 6: Simulation and experiment for the GPS antenna return loss.

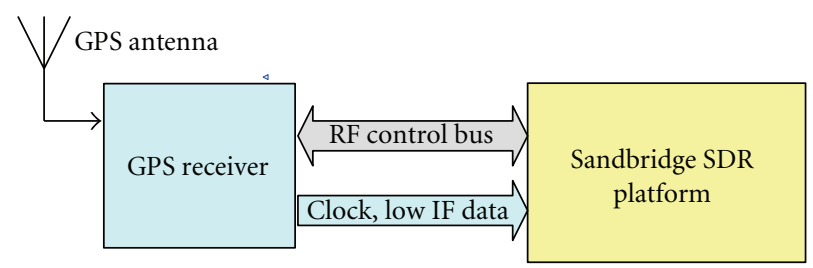

FIGURE 7: GPS test platform.

second on common PCB substrate: $1.5 \mathrm{~mm}$ thick, $18.5 \mathrm{~mm}$ wide, $33 \mathrm{~mm}$ high, and $\varepsilon_{r}=4.6$. For the antenna shown in Figure 5 the values of $\left(d_{i}\right)$ and $\left(w_{i}\right)$, measured in $\mathrm{mm}$, (as shown in Figure 1) are presented in Table 1.

We tested the antenna performance first in the lab. After the lab performance test the overall performance was evaluated on real GPS receiver. Figure 6 illustrates both the simulation and test results for the PCB substrate GPS antenna. From Figure 6 it can be seen that the return loss $S_{11}$ is $-25.77 \mathrm{~dB}$ at $1.5753 \mathrm{GHz}$. The measured bandwidth at $1.5753 \mathrm{GHz}$ is around $4 \mathrm{MHz}$ while at $S_{11}=-10 \mathrm{~dB}$ the bandwidth is $67 \mathrm{MHz}$.

The field test for the antenna was performed on the Sandbridge Technology SDR platform as described in Figure 7. The total sensitivity of the GPS receiver with the passive antenna was estimated at $-132 \mathrm{dBm}$ [23] while adding a $27 \mathrm{~dB}$ signal booster right after the antenna, the total sensitivity came down to $-159 \mathrm{dBm}$.

4.2. PIN Diode Multiband Antenna. The composite folded dipole antenna can be electronically tuned in frequency by adding a PIN diode to the matching circuit on the active layer as shown in Figure 8. For the prototype antenna we used the same RT-6010LM substrate and physical dimensions $17 \times$ $22 \times 2.45 \mathrm{~mm}$. The antenna configuration is presented in Figure 8 . 
TABLe 1: $d_{i}$ and $w_{i}$ values, measured in mm, for GPS antenna.

\begin{tabular}{cccccccccccccc}
\hline$i$ & 0 & 1 & 2 & 3 & 4 & 5 & 6 & 7 & 8 & 9 & 10 & 11 \\
\hline$d_{i}$ & 22 & 17.6 & 10.6 & 2.0 & 2.0 & 1.5 & 3.1 & 2.0 & 1.5 & 1.5 & 20.8 & 20.8 & 1.0 \\
$w_{i}$ & 15 & 7.1 & 1.4 & 0.5 & 5.8 & 0.5 & 0.5 & 1.5 & 2.5 & - & - & - & - \\
\hline
\end{tabular}

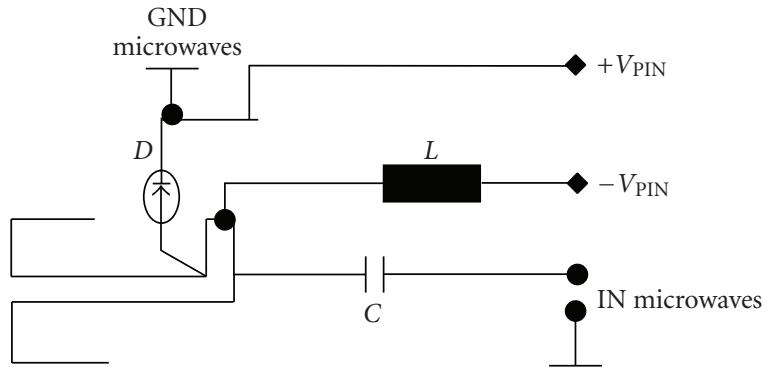

FIgURe 8: PIN diode antenna diagram. $D$ is the PIN diode, $L=$ $0.2 \mathrm{mH}$, and $\mathrm{C}=40 \mathrm{pF}$.

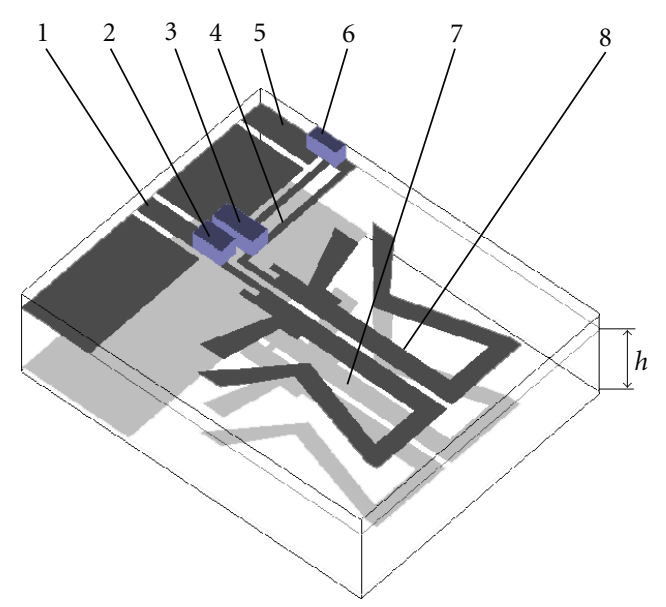

FIgURE 9: Antenna configuration with PIN diode.

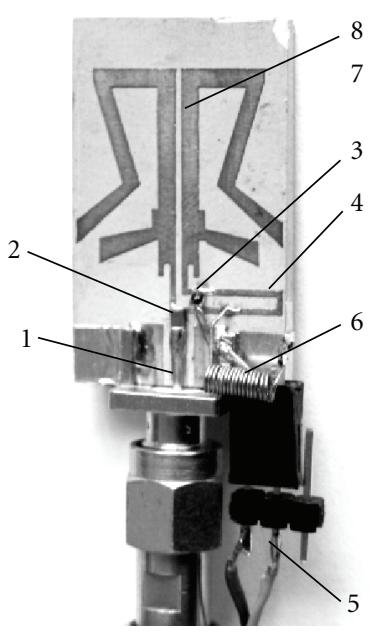

Top

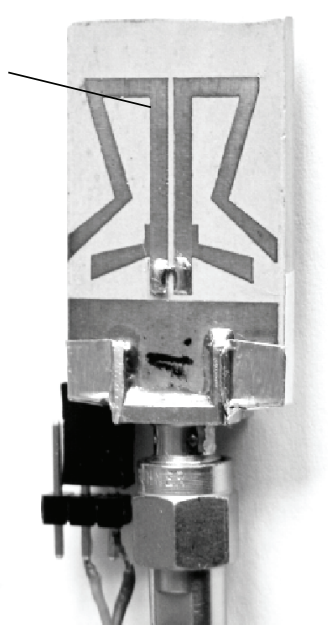

Bottom
FIgURE 10: Picture of the PIN diode antenna, top and bottom.

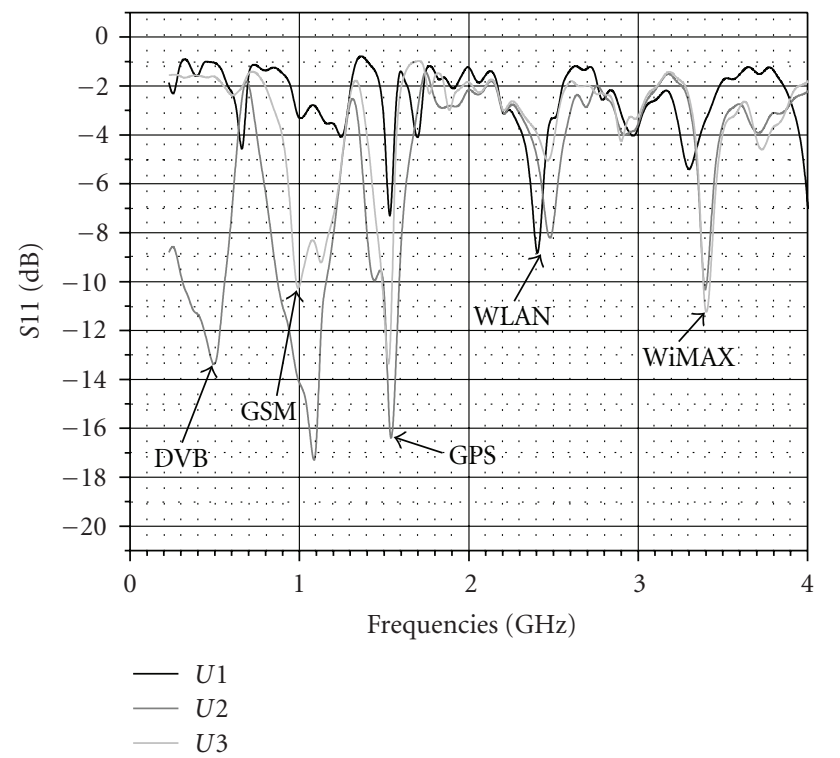

FIgURE 11: Return loss measuremen $U 1=0.0 \mathrm{~V}, U 2=0.65 \mathrm{~V}, U 3=$ $2.00 \mathrm{~V}$ are the PIN diode polarization voltages.

The input RF line (1) is DC decoupled (2) from the antenna top layer (8). The two branches of the dipole are phase shifted by a delay line (4). The PIN diode (3) does the impedance matching, frequency band selection, and it also changes the dipole polarization. The diode is tuned through the DC line (5) and the inductance (6). The bottom layer (7) of the antenna is totally separated from the top by the dielectric material of thickness $h$ as shown in Figure 9. A picture of the antenna with the PIN-DC connector is presented in Figure 10.

For the antenna shown in Figure 10 the values of $\left(d_{i}\right)$ and $\left(w_{i}\right)$, measured in $\mathrm{mm}$, (as shown in Figure 1) are presented in Table 2.

For this design configuration the return loss measurement $S_{11}$ is the main feedback parameter. Figure 11 depicts the return loss of the antenna as a function of frequency for different PIN diode polarizations, $U 1<U 2<U 3$, where $U 1=0 \mathrm{~V}, U 2=0.65 \mathrm{~V}$, and $U 3=2.00 \mathrm{~V}$. It can be seen that the return loss for different frequency bands varies with the PIN diode polarization. This property will be exploited to tune the antenna for various frequency bands required by specific communication standards.

The next diagrams illustrate the measurements performed to characterize the electric field pattern as a function of the PIN diode polarization in the frequency bands of interest. The directive gain of the antenna was measured in the XOY plan, normal to the antenna layers, as shown in Figure 12. 
TABLE 2: $d_{i}$ and $w_{i}$ values, measured in mm, for the antenna with PIN diode.

\begin{tabular}{cccccccccccccc}
\hline$i$ & 0 & 1 & 2 & 3 & 4 & 5 & 6 & 7 & 8 & 9 & 10 & 11 & 12 \\
\hline$d_{i}$ & 17 & 12.8 & 9.5 & 1.9 & 2.9 & 2.0 & 5.5 & 3.0 & 1.5 & 1.4 & 1.0 & 1.25 & 1.0 \\
$w_{i}$ & 14.8 & 7.1 & 1.4 & 0.5 & 5.8 & 0.5 & 0.5 & 1.5 & 2.5 & - & - & - & - \\
\hline
\end{tabular}

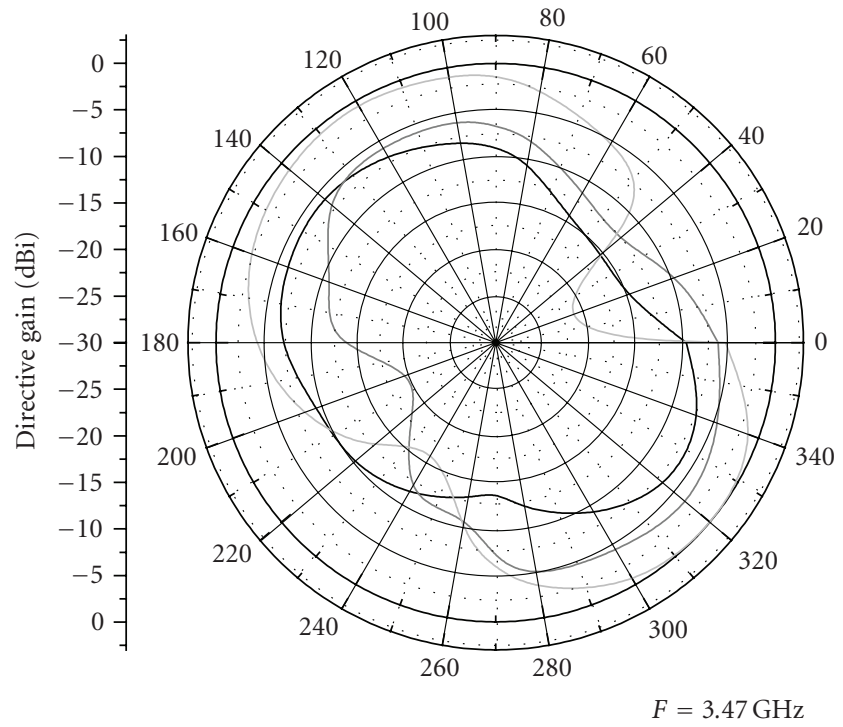

$-U 1$

$-U 2$

$-U 3$

Figure 12: Directive gain for WiMAX at $3.47 \mathrm{GHz}$.

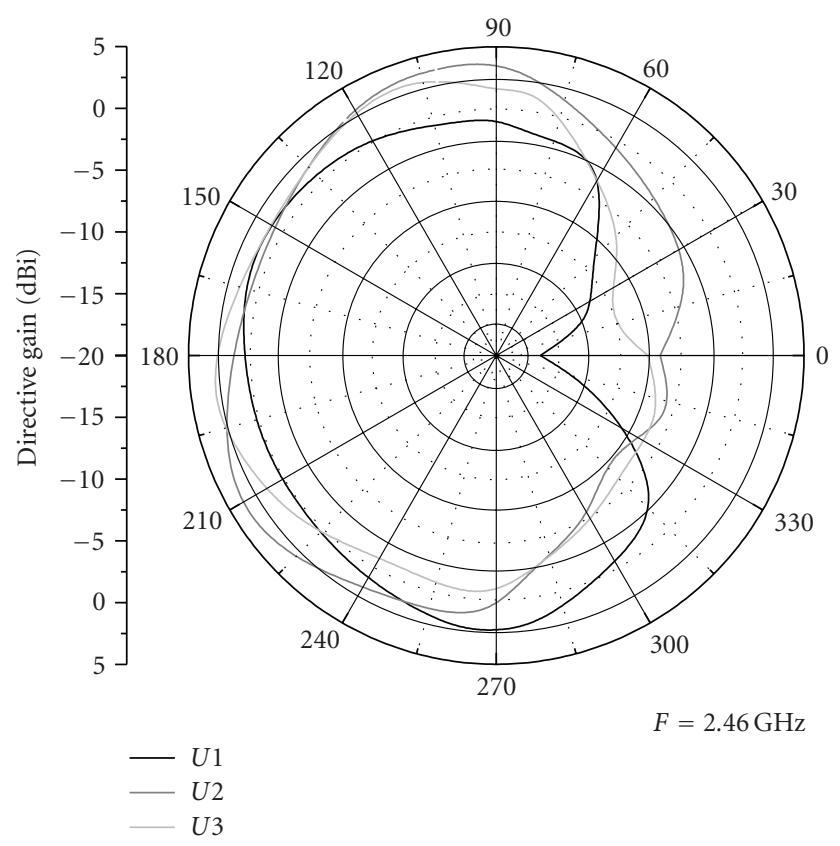

FIgURE 13: Directive gain for WLAN at $2.45 \mathrm{GHz}$.

In the WiMAX frequency band, Figure 12 shows a gain improvement of $6 \mathrm{~dB}$ corresponding to the switching of the

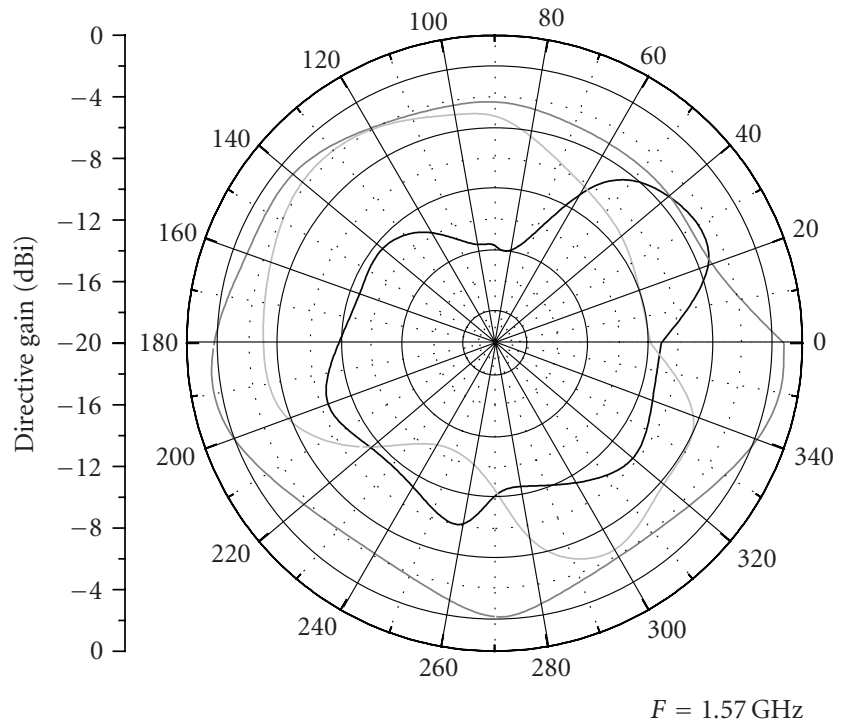

$U 1$
$U 2$
$U 3$

Figure 14: Directive gain for GPS at $1.57 \mathrm{GHz}$.

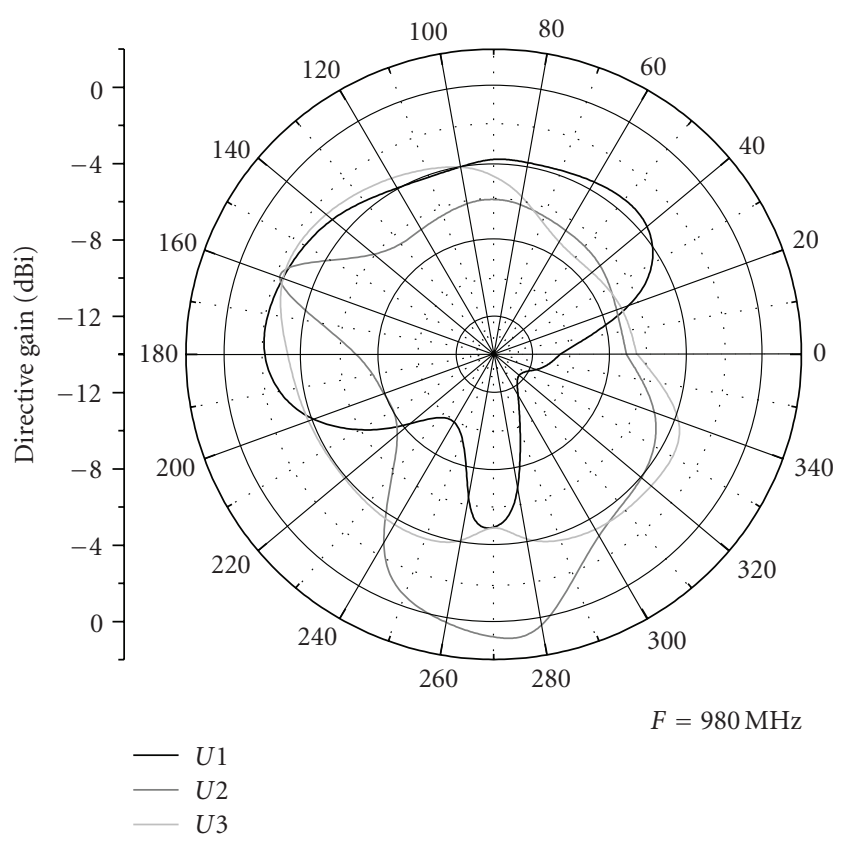

FIGURE 15: Directive gain for GSM at $980 \mathrm{MHz}$.

PIN polarization from $U 1$ to $U 3$. The main radiative direction remains unchanged at $135^{\circ}$ ( $45^{\circ}$ to the $O Y$ normal). 


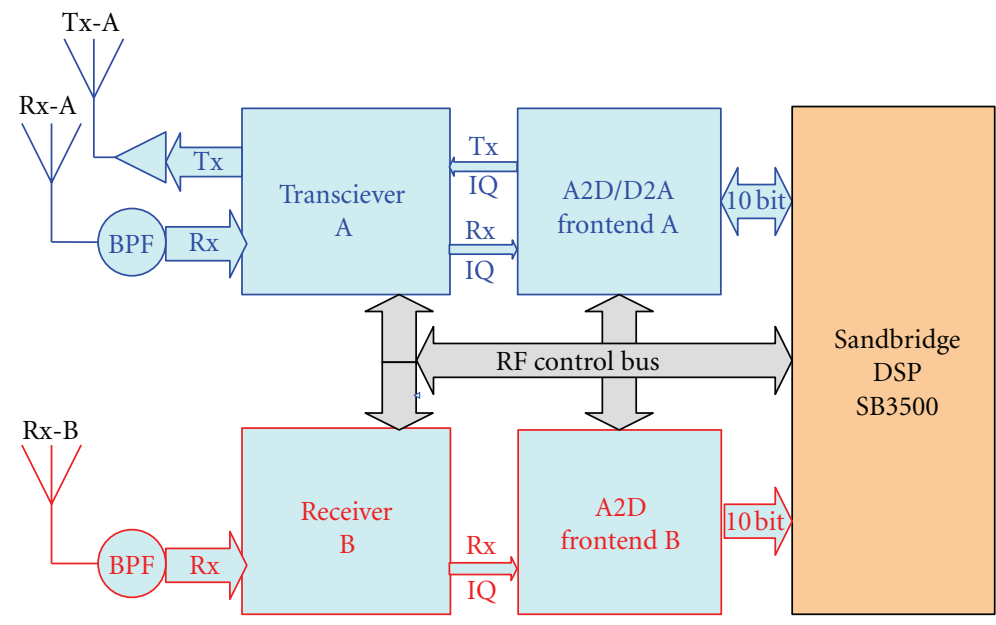

FIGURE 16: SDR platform using three antennas.

In the WLAN frequency band, the PIN diode polarization, switched from $U 1$ to $U 2$, caused an increase in the normal direction gain $\left(\Phi=90^{\circ}\right)$ from $-0.9 \mathrm{dBi}$ to $3.7 \mathrm{dBi}$ as shown in Figure 13.

In the GPS frequency band the changes with the PIN polarization voltage are more dramatic. As the polarization voltage changes from $U 1$ to $U 2$ the directivity pattern will change from directional at $35^{\circ}$ to omnidirectional. The gain rests unchanged at $35^{\circ}$, but increases by an average of $5 \mathrm{~dB}$ in the other directions (Figure 14). If the voltage is changed from $U 1$ to $U 3$ the directional pattern stays unchanged but the radiation direction will change with $90^{\circ}$ from $35^{\circ}$ to $125^{\circ}$ with a gain improvement of $1.5 \mathrm{~dB}$.

In the GSM band, a gain improvement of $6 \mathrm{~dB}$ at $270^{\circ}$ is observed when the polarization changes from $U 1$ to $U 2$ (practically a reverse in the radiation direction). Also a change from directional (at $U 1$ ) to omnidirectional pattern (at U3) can be observed (see Figure 15).

4.3. MIMO Systems. Multiple folded dipole composite antenna can be placed on the same PCB to give way to a MIMO system. Three such antennas, two on the receive side and one on the transmit side were used on the Sandbridge Technology form factor SDR platform capable of executing both WiMAX and WLAN [17].

The block diagram of the SDR platform is illustrated in Figure 16 while the picture of the card with the antennas is shown in Figure 17. The TOP layer includes the microstrip feeding point and the impedance matching circuit of each antenna in the system to $50 \mathrm{Ohms}$ line impedance. The bottom layer acts as frequency selective reflector, having similar print pattern as the top layer. In order to reduce the mutual coupling between antennas, a free space cut of $2.5 \mathrm{~mm}$ was added to the dielectric substrate near the sides of the middle antenna.

The antennas in the MIMO system were positioned such a way to maximize the spatial diversity. In order to increase the isolation between antennas, the middle antenna in the

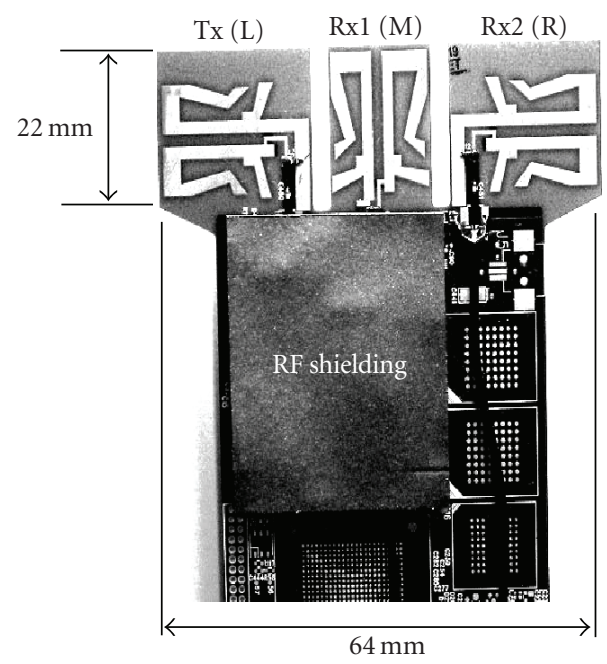

FIGURE 17: Picture of the SDR platform with three antennas.

system was positioned to have the polarization perpendicular to the lateral antennas polarization.

The antennas system was built on 12 layers on Isola FR 406BC PCB with $1.2 \mathrm{~mm}$ total thickness, global permittivity $\varepsilon_{r}=3.8$ and dissipation factor less than $\operatorname{tg} \delta=$ 0.0140 . The PCB gap between antennas is $2.5 \mathrm{~mm}$ wide.

For the MIMO antennas system described in Figure 17, the values of $\left(d_{i}\right)$ and $\left(w_{i}\right)$, measured in $\mathrm{mm}$, (as shown in Figure 1) are presented in Table $3 . Z S_{\mathrm{B}}$ is open circuit for $\mathrm{Rx} 1$ antenna and short circuit $\left(Z S_{\mathrm{B}}=0 \mathrm{Ohm}\right)$ for $\mathrm{Tx}$ and $\mathrm{Rx} 2$ antennas.

A $2 \times 2$ MIMO system, capable of both FDD and TDD communication modes, can be achieved by adding the forth antenna, as shown in Figure 18. The four-antenna system is an improved version of the previous three-antenna design, where Rx1 antenna has been symmetrically duplicated. In this case the complexity of the design will increase due to mutual coupling between the antennas. To reduce the mutual coupling a metal shield is added as shown in Figure 18. 
TABLE 3: $d_{i}$ and $w_{i}$ values, measured in $\mathrm{mm}$, for the three antennas system.

\begin{tabular}{cccccccccccccc}
\hline$i$ & 0 & 1 & 2 & 3 & 4 & 5 & 6 & 7 & 8 & 9 & 10 & 11 & 12 \\
\hline$d_{i}$ & 17 & 11.0 & 10.5 & 1.2 & 2.8 & 2.0 & 3.8 & 2.4 & 1.5 & 2.4 & 1.25 & 0.7 & 1.0 \\
$w_{i}$ & 14 & 4.5 & 1.5 & 1.0 & 5.0 & 1.5 & 0.5 & 1.5 & 0.5 & - & - & - & - \\
\hline
\end{tabular}

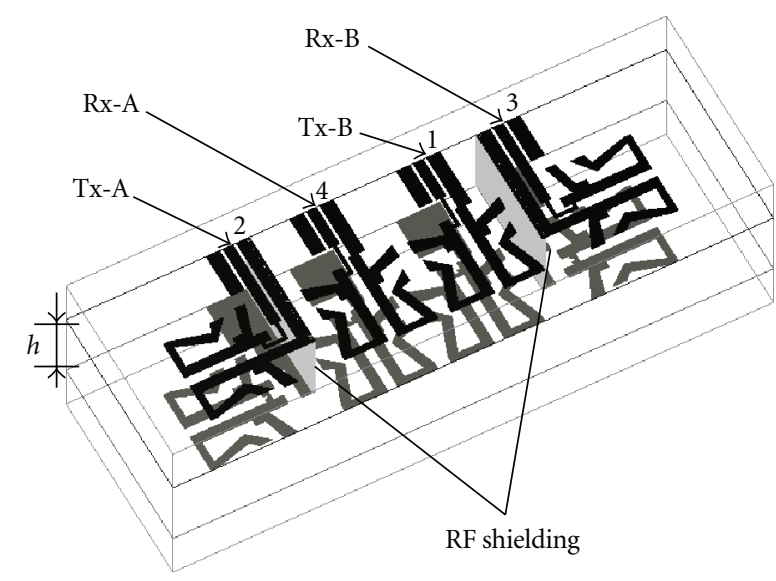

FIGURE 18: Four antennas in $2 \times 2$ MIMO configuration.

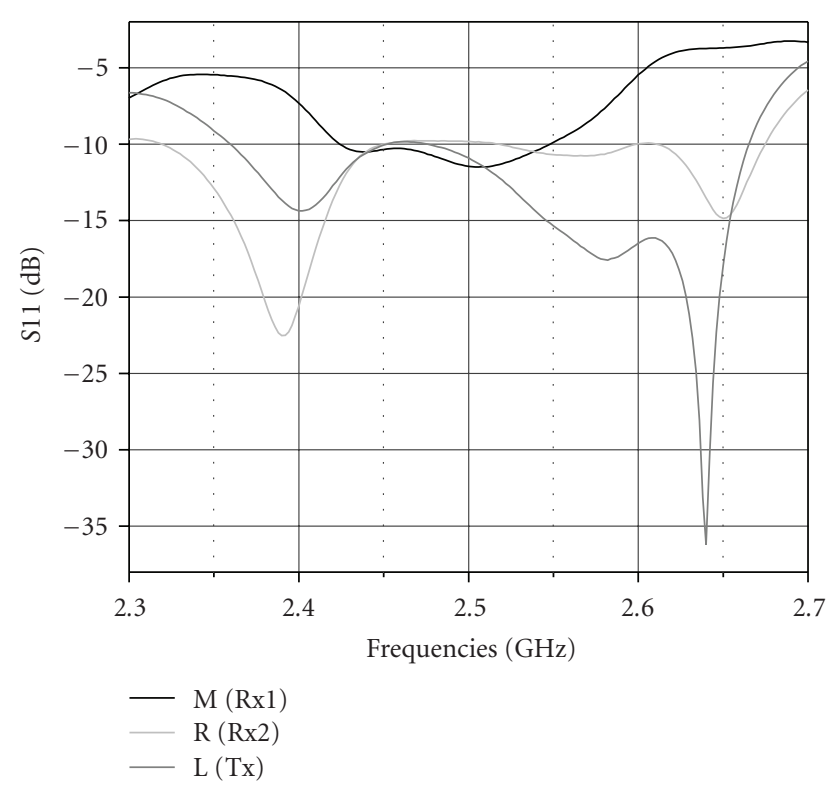

FIGURE 19: Return loss $S_{11}$ measurements for MIMO system.

Two types of measurement were performed for the MIMO antennas system characterizations: return loss $S_{11}$ and isolation $S_{i j}$ between antennas. The return loss measurements are illustrated in Figure 19 for the MIMO system of three antennas.

In Figure 19 it can be seen that the return loss $S_{11}$ for the $\mathrm{M}$ and $\mathrm{R}$ (Figure 17) antennas is less than $-5 \mathrm{~dB}$ in the $2.3-2.6 \mathrm{GHz}$ band. The best antenna in the system is the Tx (L in Figure 17) antenna with $S_{11}>-10 \mathrm{~dB}$ in the 2.3-2.67 GHz band. In the WLAN band the standing wave ratio is less than $-10 \mathrm{~dB}$ for all antennas.

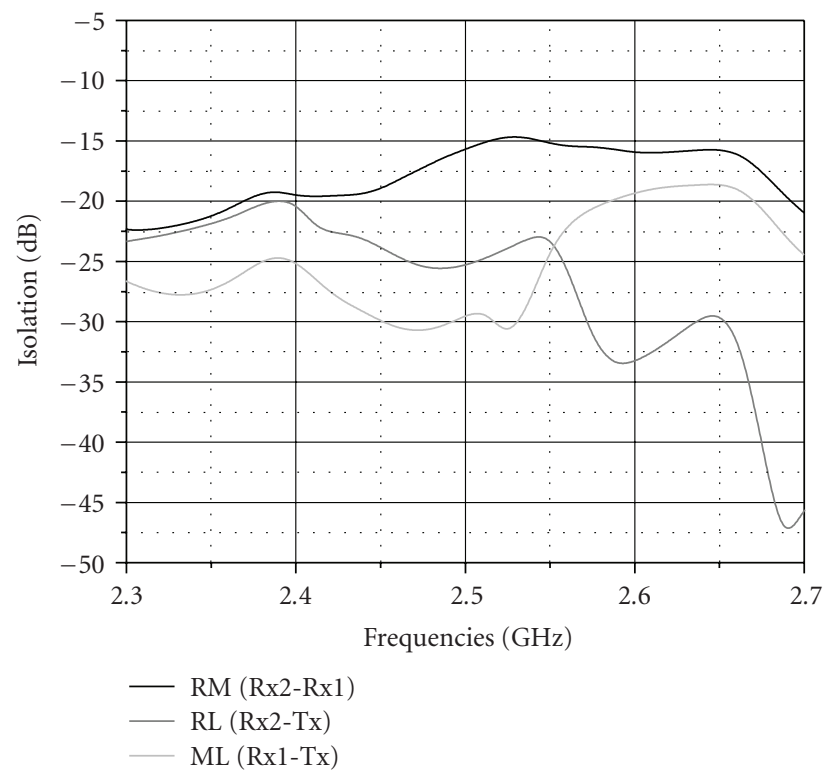

FIGURE 20: Isolation between antennas for the three-antenna configuration.

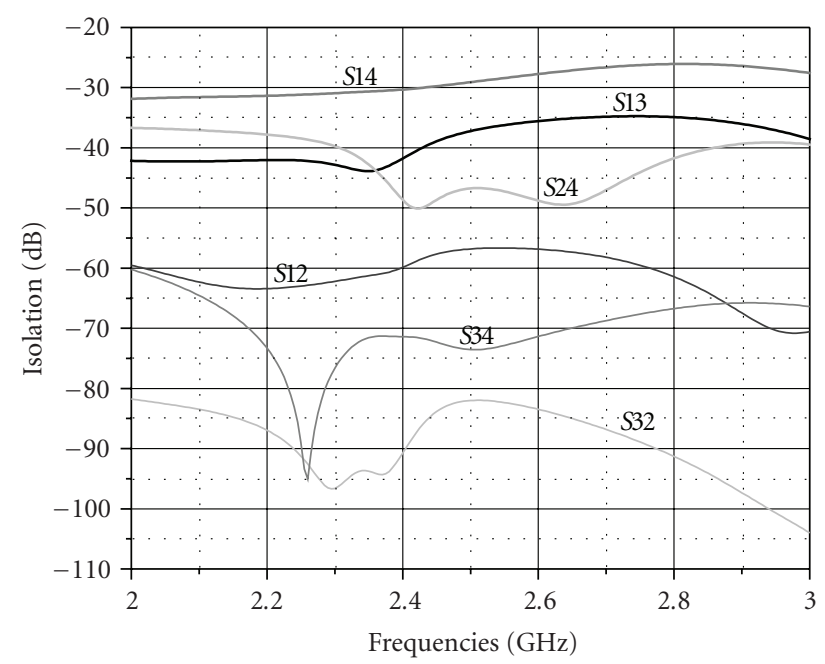

Figure 21: Isolation between antennas for the four-antenna configuration.

We estimated the mutual coupling by using one of the antennas as a transmit antenna and the other two as receive antennas. The isolation measurements between antennas are presented in Figure 20. It can be seen an isolation better than $-15 \mathrm{~dB}$ between all antennas.

Theoretical analyse of the $2 \times 2$-antenna configuration regarding the isolation between antennas show that the worst isolation is between antennas 1 and 4 and, antennas 1 and 3 as shown in Figure 21. 


\section{Conclusions}

We have shown that starting from a basic antenna design there are many possibilities for practical configurations targeting mobile applications. A single composite antenna can be configured to be a directional or omnidirectional GPS antenna. With a few minor changes in the shape of the print and adding an adaptive/tuning circuit with a PIN diode can significantly improve the antenna gain, polarization, and selectivity proprieties. A shift and enlargement of the frequencies bands has also been observed. From directivity diagrams follows that the antenna directive gain depends on the PIN diode polarization. It can also be observed an improvement in the antenna gain and a change in the antenna radiation pattern distribution. We proved that by changing the PIN diode DC voltage it is possible to select a specific directivity and also to select a number of simultaneous frequency bands or to enlarge one or more frequency bands.

We also showed that starting from the single antenna configuration a system of MIMO antennas can be also obtained with very good performance.

\section{References}

[1] J. Glossner, M. Schulte, M. Moudgill, et al., "Sandblaster lowpower multithreaded SDR baseband processor," in Proceedings of the 3rd Workshop on Applications Specific Processors (WASP '04), pp. 53-58, Stockholm, Sweden, September 2004.

[2] C. Sturm, M. Porebska, J. Timmermann, and W. Wiesbeck, "Investigations on the applicability of diversity techniques in ultra wideband radio," in Proceedings of the International Conference on Electromagnetics in Advanced Applications (ICEAA '07), pp. 899-902, Torino, Italy, September 2007.

[3] A. Lambrecht, S. Schulteis, and W. Wiesbeck, "Diversity antenna system for radio reception in automative applications," in Proceedings of the International Conference on Electromagnetics in Advanced Applications (ICEAA '07), pp. 21-24, Torino, Italy, September 2007.

[4] G. Jue and Agilent Technologies, "Addressing the design and verification challenges of long term evolution," Wireless Design \& Development, no. 9, pp. 24-30, 2008.

[5] E. Surducan, D. Iancu, and J. Glossner, "Modified printed dipole antenna for wireless multi-band communication devices," in Proceedings of the URSI International Symposium on Electromagnetic Theory (EMTS '04), pp. 1161-1163, Pisa, Italy, May 2004.

[6] E. Surducan, D. Iancu, and J. Glossner, "Modified printed dipole antennas for wireless multi-band communication systems," US patent no. 7034769, April 2006.

[7] E. Surducan, D. Iancu, and J. Glossner, "Modified printed dipole antennas for wireless multi-band communication systems," US patent no. 7095382, April 2006.

[8] R. Garg, P. Bhartia, I. Bahl, and A. Ittipibonon, Microstrip Antenna Design Handbook, Artech House, London, UK, 2001.

[9] E. L. Krenz and D. J. Tammen, "Single compact dual mode antenna," US patent no. 5532708, July 1996.

[10] E. Surducan, D. Iancu, V. Surducan, and J. Glossner, "Microstrip composite antenna for multiple communication protocols," International Journal of Microwave and Optical Technology, vol. 1, no. 2, pp. 772-775, 2006.
[11] E. Surducan, D. Iancu, and J. Glossner, "Microstrip multiband composite antenna," patent pending WO/2006/086194, August 2006.

[12] R. Gonzalo, G. Nagore, and P. de Maagt, "Simulated and measured performance of a patch antenna on a 2-dimensional photonic crystals substrate," Progress in Electromagnetics Research, vol. 41, pp. 257-269, 2003.

[13] H. Mosallaei and K. Sarabandi, "Engineered meta-substrates for antenna miniaturization," in Proceedings of the URSI International Symposium on Electromagnetic Theory (EMTS '04), vol. 1, pp. 191-193, Pisa, Italy, May 2004.

[14] N. Kaneda, W. R. Deal, Y. Qian, R. Waterhouse, and T. Itoh, "A broad-band planar quasi-Yagi antenna," IEEE Transactions on Antennas and Propagation, vol. 50, no. 8, pp. 1158-1160, 2002.

[15] E. Surducan, D. Iancu, V. Surducan, and S. Stanley, "Multiband antennas for SDR wireless handset application," in Proceedings of the International Conference on Electromagnetics in Advanced Applications (ICEAA '07), pp. 523-526, Torino, Italy, September 2007.

[16] C. B. Dietrich Jr., R. M. Barts, W. L. Stutzman, and W. A. Davis, "Trends in antennas for wireless communications," Microwave Journal, vol. 46, no. 1, pp. 22-44, 2003.

[17] E. Surducan, D. Iancu, V. Surducan, and J. Glossner, "Miniature multiband antennas for hand held WiMAX and WiFi application," in Proceedings of the International Conference on Electromagnetics in Advanced Applications (ICEAA '07), pp. 13-16, Torino, Italy, September 2007.

[18] F. Yang and Y. Rahmat-Samii, "A reconfigurable patch antenna using switchable slots for circular polarization diversity," IEEE Microwave and Wireless Components Letters, vol. 12, no. 3, pp. 96-98, 2002.

[19] H.-R. Chuang, L.-C. Kuo, C.-C. Lin, and W.-T. Chen, "A $2.4 \mathrm{GHz}$ polarization-diversity planar printed dipole antenna for WLAN and wireless communication applications," Microwave Journal, vol. 45, no. 6, pp. 50-62, 2002.

[20] E. Surducan, V. Surducan, D. Iancu, and J. Glosner, "Multiband antenna with adaptive circuit," in Proceedings of the 11th International Symposium on Microwave and Optical Technology (ISMOT'07), pp. 633-637, Villa Mondragone, Italy, December 2007.

[21] M. D. Foegelle, "Antenna pattern measurement: concepts and techniques," Compliance Engineering, vol. 19, no. 3, pp. 22-33, 2002.

[22] M. D. Foegelle, "Antenna pattern measurement: theory and equations," Compliance Engineering, vol. 19, no. 3, pp. 34-43, 2002.

[23] M/A-COM, "Selection of antenna and cable for optimum automotive GPS performance," Application note M541, V4.0. 

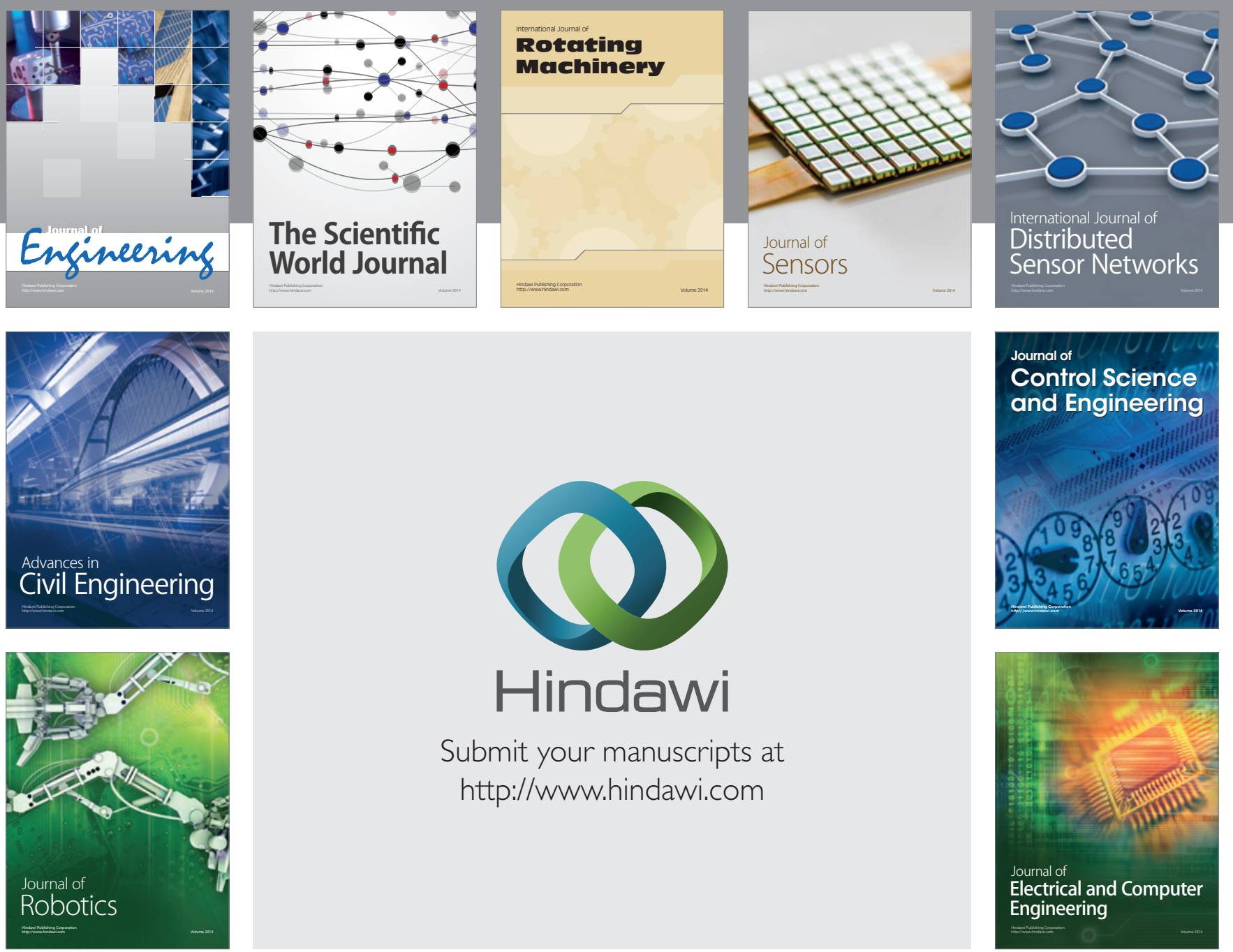

Submit your manuscripts at

http://www.hindawi.com
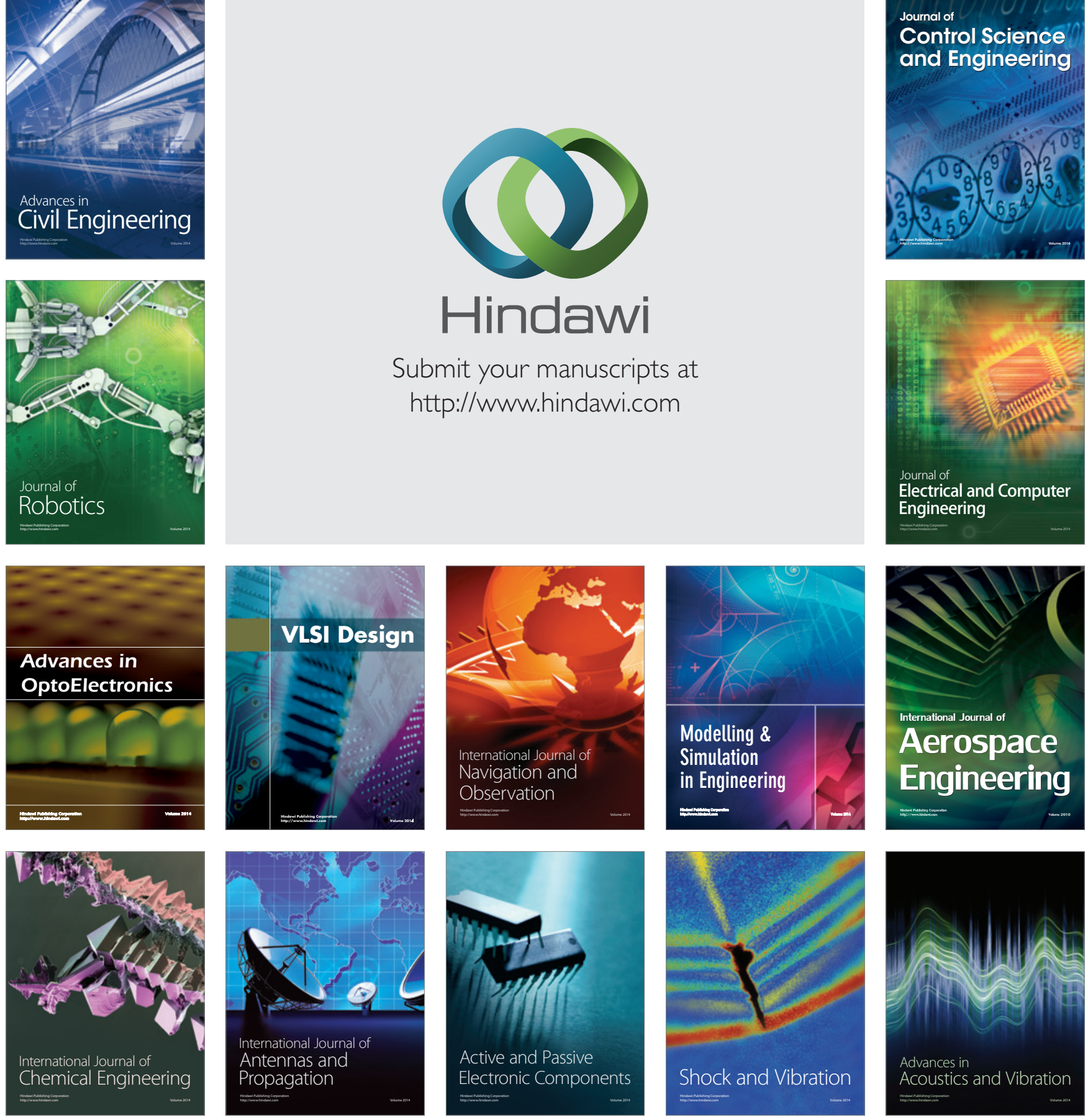